\title{
Sleep reduces haematopoiesis and atherosclerosis via a neuroimmune axis
}

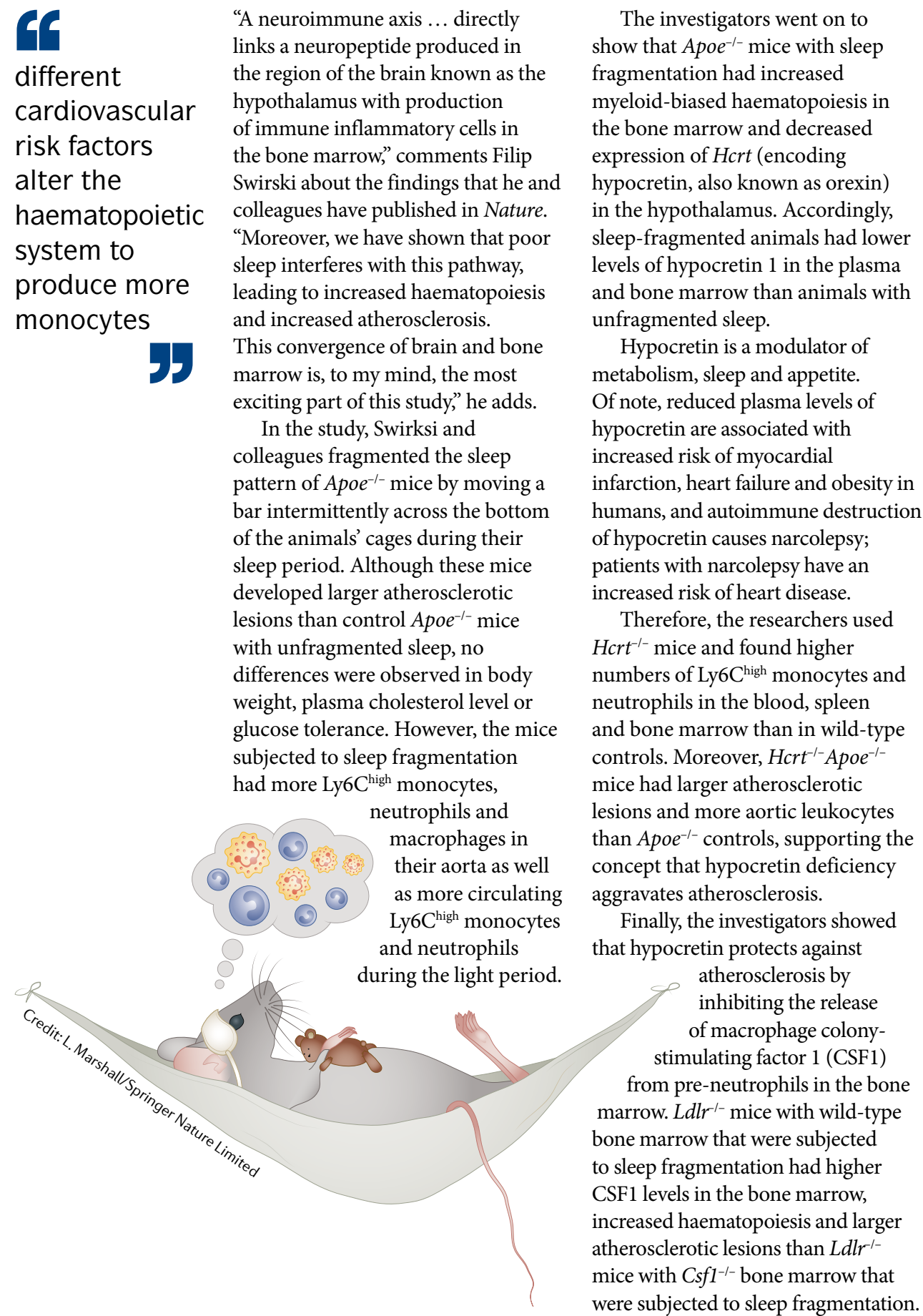

Interestingly, $A p o e^{-/-}$mice subjected to sleep fragmentation that received hypocretin 1 delivered to the periphery had reduced numbers of circulating monocytes and neutrophils, lower levels of CSF1 in the bone marrow and smaller atherosclerotic lesions compared with control animals.

Haematopoiesis is emerging as one of the most important processes in the body in the pathogenesis of cardiovascular disease. "[The findings by Swirski and colleagues] contribute to a larger body of work showing how different cardiovascular risk factors alter the haematopoietic system to produce more monocytes," comments Andrew Murphy from the Baker Heart and Diabetes Institute in Melbourne, Australia, and who was not involved in the study. "There is a rationale to directly target the proliferation or lineage selection of the haematopoietic system to tone down the production of monocytes, neutrophils and platelets in people at high risk of a cardiovascular event, but this should be met with caution as we do not want to put patients at risk when the body requires the haematopoietic system to respond, especially in acute situations like infection," warns Murphy. Swirksi and colleagues are now collaborating with clinicians to test this novel neuroimmune pathway in humans.

Gregory B. Lim

ORIGINAL ARTICLE McAlpine, C. S. et al. Sleep modulates haematopoiesis and protects against atherosclerosis. Nature 566, 383-387 (2019)

FURTHER READING Tobaldini, E. et al. Short sleep duration and cardiometabolic risk: from pathophysiology to clinical evidence. Nat. Rev. Cardiol. https://doi.org/10.1038/s41569-0180109-6 (2018) 